\title{
Accurate and High Quality Triangle Models from 3D Grey Scale Images
}

\author{
P.W. de Bruin ${ }^{1}$, P.M. van Meeteren ${ }^{2}$, F.M. Vos ${ }^{2}$, A.M. Vossepoel ${ }^{2}$, and F.H. Post ${ }^{1}$ \\ ${ }^{1}$ Computer Graphics, Faculty of Information Technology and Systems, \\ Delft University of Technology \\ \{P.W.deBruin,F.H.Post\}@its.tudelft.nl \\ 2 Pattern Recognition Group, Department of Applied Physics, Delft University of Technology \\ \{magchiel,frans, albert\}@ph.tn.tudelft.nl
}

\begin{abstract}
Visualization of medical data requires the extraction of surfaces that represent the boundaries of objects of interest. This paper describes a method that combines finding these boundaries accurately and ensuring that this surface consists of high quality triangles. The latter is important for subsequent visualization and simulation. We show that the surfaces created using this method are both accurate and have good quality triangles.
\end{abstract}

\section{Introduction}

Analysis of $3 \mathrm{D}$ medical images is aided by creating $3 \mathrm{D}$ surface representations that describe the boundaries of anatomical structures. A triangle mesh facilitates viewing and manipulating the data easily. Apart from that, these meshes can be used to calculate metrics on its size and shape. Applications include computer aided diagnosis, surgical planning, and simulation.

Extracting a mesh, or segmenting data, requires detecting and grouping of parts in the data that share certain characteristics. A straightforward method to segment data is isosurfacing (e.g., Marching Cubes [12]), where points that share the same greyscale value (the isovalue) are assumed to describe the surface. However, this assertion does not always hold due to noise and bias (e.g., in Magnetic Resonance Imaging).

Another approach to segmenting is to identify objects by an edge: an abrupt change in greyscale intensity. By doing so, the exact greyscale value of the points on the boundary is not relevant. Edge detection is also hampered by noise due to the sensitivity of the derivative operator to high frequency noise. Therefore, a Gaussian derivative is often used to suppress noise. Unfortunately, the reduction of noise coincides with dislocation of edges. A good trade-off is achieved between detecting and locating edges and suppressing noise by using a scale-space approach $[1,7]$.

The quality of an extracted mesh is important for subsequent processing steps, such as visualisation and finite element modeling methods. Here good quality triangles and a smooth mesh and are preferred and sometimes even required (see Section 2.3). Surface extraction methods such as deformable models $[13,15]$ and snakes $[11,14]$ do not explicitly attempt to produce meshes of good triangle quality. Apart from that, deformable models and snakes require several parameters that have to be tuned for each case. Therefore, it is difficult to maintain reproducibility. The SurfaceNets method does take triangle quality into account, but is limited to isosurfaces $[6,10]$. The conventional approach is to proceed after extraction by applying mesh improvement techniques. However, this 

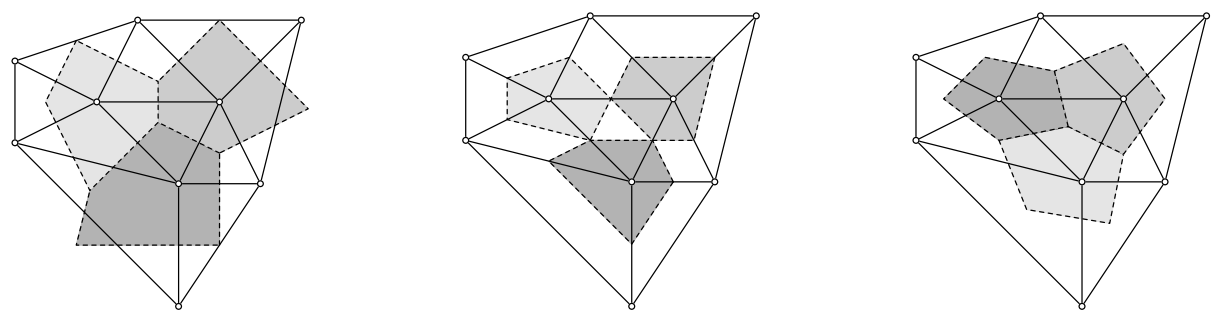

Fig. 1. Constraining regions based on (from left to right) Voronoi, edge midpoint and centroid criterion. The triangulation does not meet the Delaunay criterion, hence the odd shape. The edge midpoint does not cover the entire surface. The centroid method is insensitive to the Delaunay criterion and fills the entire mesh area.

is an undesirable situation because such techniques are purely geometrical and topological methods. The connection is lost between the mesh and the original data.

Summarizing, a good mesh extraction method should meet requirements concerning accuracy (edge detection), reproducibility (number of parameters), and smoothness and triangle quality of the generated mesh.

In this paper we present a new method that produces meshes that fulfill these criteria and does not lose the connection with the greyscale data. Our main contribution is the combination of two types of methods: multi-scale edge detection and mesh improvement. We will discuss several novel adaptations of the techniques used, and we show how the number of user-specified parameters is kept to a minimum.

In the next Section first each of the techniques indicated above is explained, followed by our method of applying these techniques. Section 3 shows the results of our method when applied to synthetic and to medical data. Conclusions and future research can be found in Section 4.

\section{Methods}

To achieve the goals and criteria outlined in Section 1 a two-fold approach is used in which high quality scale-space edge detection and mesh improvement methods are combined. Our approach consists of alternating between edge-detection and mesh improvement until a desired result is obtained. Each of the separate methods is explained below, followed by aspects of the combination.

\subsection{Scale-space edge detection}

Edge detection based on image gradients is the identification of intensity changes between a region of interest and its surroundings. The modulus of the first derivative of an image has a local maximum at locations corresponding to edges. However, highfrequency noise will yield local maxima as well. Therefore, noise suppression is necessary. A common solution to this problem is to apply a low-pass filter to the image. Although this suppresses noise, it dislocates and suppresses edges also. The Gaussian kernel optimises the criteria of good signal-to-noise ratio and good localisation simultaneously [5]. The parameter $\sigma$ of the Gaussian kernel determines the level of smoothing that is applied to the image.

Scale-space theory embeds an image in a one-parameter family of derived images: the scale space $[7,16]$. A stack of images is created by convolving the original image with increasingly smoothing Gaussian derivative kernels. 
The image corresponding to the largest value for $\sigma$ is severely blurred, but noise is suppressed. In this image the detection of edges is a simpler task. Once an edge is detected, the found location is taken as the initial position in the image corresponding to the next lower value for $\sigma$. This process continues until the lowest $\sigma$ is reached. At this point the edge is localised completely.

In order to limit the search space a simple representation of the object to extract is required. This coarse segmentation does not need to be accurate but an estimate of the maximum distance from the mesh to the real object is required. The search for edges is limited to the region indicated by the mesh and the maximum distance.

The following three important points have to be addressed: 1) selecting the upper and lower bound of $\sigma, 2$ ) sampling the scale space, and 3) locating the edge (selection of maximum).

The upper and lower bound $\sigma$ selection is important because not every scale is relevant. The upper bound effectively determines the region of capture of the edge detector and is set equal to the maximum deviation of the initial mesh to the real object. The lower bound of $\sigma$ is set equal to voxel length.

Next, a discrete set of $\sigma$ 's between the upper and lower bound of $\sigma$ is calculated. A straightforward linear sampling is not appropriate because this might lead to aliasing at fine scales and over-sampling at coarse levels of scale. We have chosen for a logarithmic sampling method described by $\sigma_{i}=\sqrt[n]{2^{i}}$ [?]. The number $n$ determines how many times $\sigma$ is sampled per doubling of the scale parameter. By doing so, a proper and intuitive sampling is obtained because a large $\sigma$ corresponds to a large stepsize and a small $\sigma$ corresponds to a small stepsize.

At every vertex in the initial mesh the grey scale gradient vector is calculated. Along this vector the search for the edge is performed. The search window is made scalerelative to avoid distraction by spurious edges. The total width of the search window is set equal to $2 \sigma$. At five points along the line the modulus of the gradient is calculated with step size equal to $0.5 \sigma$. The point corresponding to the maximum value of the five points is the starting point in the next lower scale. Sampling the search window at 5 discrete positions introduces an uncertainty of half the step size. Because the lowest scale we encounter is $\sigma=1$, the localisation will have an uncertainty equal to 0.25 voxel lengths. When going from one scale to the next lower the new search window is placed centered at the found maximum. If the new window exceeds the boundaries, it is translated along the gradient vector until it is completely located inside the region of capture. Without this correction the search could move outside the region of capture of the detector.

\subsection{Mesh improvement techniques}

Mesh improvement techniques operate either on the geometry or the topology to improve the quality of a mesh [8] (for measures of mesh quality see Section 2.3). Geometric methods reposition vertices, whereas topological methods operate on the connections between the vertices. For our work we have chosen one of each type: Laplacian smoothing (geometric) and edge swapping (topological).

The Laplacian smoothing operator $S_{\mathrm{L}}$ (see e.g., [8]) moves each vertex $\vec{v}_{i}$ of a mesh to the average position of its $N$ linked neighbour vertices $\vec{v}_{j}$ by

$$
\overline{\vec{v}}_{i}^{L}=\mathrm{S}_{\mathrm{L}}\left(\vec{v}_{j}\right)=\frac{1}{N} \sum_{j=1}^{N} \vec{v}_{j}, \quad j \neq i
$$


The position of each vertex in the mesh is updated after all new positions are calculated.

Advantages of Laplacian smoothing are that it is a computationally inexpensive operation, it produces a mesh with a smooth surface, and it does not require any parameters to tune. Disadvantages of this method are that it does not guarantee an increase of the triangle quality (e.g., vertices placed symmetrically around a vertex), it shrinks the mesh, converges slowly, and it can introduce geometric errors.

A geometric error is an inversion of the triangle orientation, which is detected by an change in direction of the triangle normal (e.g., pointing outward from a closed object surface). To prevent geometric errors a region of constrained movement for each vertex is created. These regions should be mutually exclusive and collectively exhaustive. By doing so, the region of movement for each vertex is as large as possible without the possibility of geometric errors. Figure 1 shows three constraining regions. The first is a Voronoi region. Here, the regions are constructed by connecting the regions created by a Delaunay triangulation (i.e., the circumcircle of each triangle is an empty circle). The figure shows that if the mesh does not meet the Delaunay criterion the regions overlap. The second idea is to connect the midpoints of the edges as is shown in Figure 1. The regions do not overlap, but do not cover the entire surface area either. We propose a new method where we create an allowable region of movement for each vertex by connecting the centroids of the triangles surrounding the vertex. This method covers the entire mesh surface and the regions are mutually exclusive.

Our centroid smoothing method $\mathrm{S}_{\mathrm{C}}$ prevents geometric errors and is expressed as a weighted sum of standard Laplacian smoothing $S_{\mathrm{L}}$ and the current vertex $\vec{v}_{i}$

$$
\overline{\vec{v}}_{i}^{C}=\mathrm{S}_{\mathrm{C}}\left(\vec{v}_{i}, \vec{v}_{j}\right)=\frac{1}{3} \vec{v}_{i}+\frac{2}{3} \mathrm{~S}_{\mathrm{L}}\left(\vec{v}_{j}\right)
$$

Edge swapping is a technique to improve the quality of the triangles in a mesh. Each pair of triangles sharing an edge is considered as a quad with the edge as a diagonal. According to a criterion the edge is swapped, or not.

Here, the edge swapping algorithm proposed by [9] and improved by [4] is used. The algorithm improves the regularity of a mesh. For each quad in the mesh a relaxation index $R_{i}$ is calculated that depends on the connectivity number of each vertex. Let the degree of the four vertices be $d_{1}, d_{2}, d_{3}$, and $d_{4}$, then $R_{i}$ is defined by $R_{i}=\left(d_{1}-6\right)^{2}+\left(d_{2}-6\right)^{2}+\left(d_{3}-6\right)^{2}+\left(d_{4}-6\right)^{2}$. An edge is swapped if the relaxation index after swapping is closer to zero. This method actively creates vertices with connectivity number 6, which allows all the incident angles to be close to 60 degrees.

\subsection{Quality measures}

The edge detector places vertices on the edge with an a priori known inaccuracy of $0.25 \sigma$. The inaccuracy of a mesh is defined by the distance from the centroid of each triangle to the surface as defined by the edge detector, averaged over all triangles.

There is no generally agreed definition of mesh quality; the quality measure depends on the subsequent use of the generated mesh. For specific cases quality indicators can be derived based on the approximation function and a known target. More generally, the consensus appears to be that a good quality mesh consists of triangles with not too small and not too large angles. Apart from that, meshes with regular or smoothly varying elements are "visually pleasing" [3].

A simple approach is to define the equilateral triangle as the highest quality triangle. Straightforward expressions of the quality of a triangle are the ratio of the shortest 


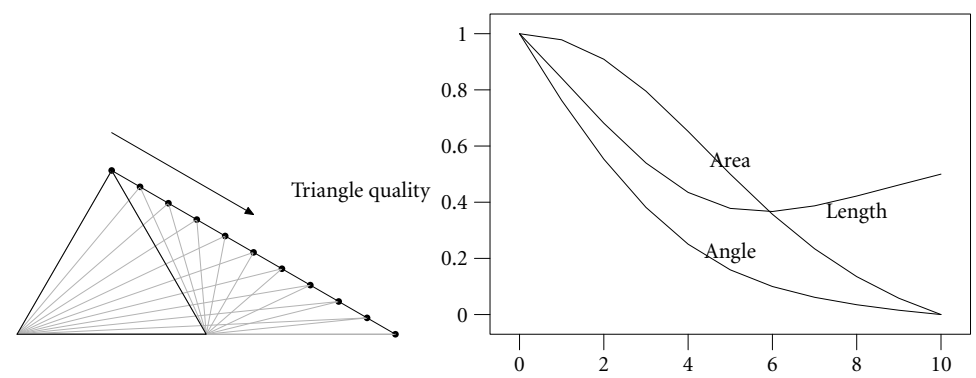

Fig. 2. A comparison of three triangle quality criteria. Starting with an equilateral triangle the top vertex is moved along the line indicated by the arrow until the triangle is degenerate. At each point along the line the quality is calculated and displayed in the graph.

edge to the longest edge and the ratio of the smallest to the largest angle. Both criteria define the equilateral triangle as the perfect triangle with quality 1 (one). However, it is required that an ideal mesh (with average quality 1) consists of equal-area triangles. Therefore, a third criterion is considered that weighs the area $A$ of the triangle in the quality measure [2] (the area of a unit equilateral triangle is $\sqrt{3} / 4$. The criteria are:

$$
q_{\text {length }}=\frac{\min \left(\left|\vec{e}_{1}\right|,\left|\vec{e}_{2}\right|,\left|\vec{e}_{3}\right|\right)}{\max \left(\left|\vec{e}_{1}\right|,\left|\vec{e}_{2}\right|,\left|\vec{e}_{3}\right|\right)}, \quad q_{\text {angle }}=\frac{\min (\alpha, \beta, \gamma)}{\max (\alpha, \beta, \gamma)}, \quad q_{\text {area }}=\frac{4 \sqrt{3} A}{\left|\vec{e}_{1}\right|^{2}+\left|\vec{e}_{2}\right|^{2}+\left|\vec{e}_{3}\right|^{2}}
$$

where edges are indicated by $\vec{e}_{i}$, angles are represented by $\alpha, \beta$ and $\gamma$ and $A$ denotes the area of the triangle.

Figure 2 shows a graphical representation of the behaviour of the quality functions in a specific situation. Clearly, the $q_{\text {length }}$ criterion is not well behaved, because for increasingly lower quality triangles the criterion outcome increases. The $q_{\text {angle }}$ and $q_{\text {area }}$ criteria are well behaved because they are bijective and monotous mappings. We have chosen the $q_{\text {area }}$ criterion for our experiments.

\subsection{Protocol}

We have described three processes that are necessary to extract a mesh with accuracy and quality. Here we describe the protocol consisting of successive applications of each of the methods. The consecutive steps in the method are: 1) Edge detection (ED), 2) Edge swap (ES), 3) (Multiple) improvement steps (S), 4) repeat steps 1,2, and 3 until desired quality is reached, and 5) a final ED and ES.

The first step should be an edge detection step to ensure that all the vertices are positioned on the boundary. Next, we proceed by regularising the mesh using the edge swap. We have found in our experiments that smoothing steps after an edge swap converge faster than starting without an edge swap. Subsequently, one or more modified Laplacian smoothing steps are applied, followed by and edge detection step and edge swap. The region of capture of the edge detector is known and, therefore, several smoothing steps can be applied to achieve faster mesh improvement. The distance that each vertex travels from its initial position is calculated and if this distance exceeds the region of capture of the edge detector the next cycle is started. When the quality of the triangles has reached a desired value the sequence is ended with an edge detection step and an edge swap to ensure that each vertex is on the boundary. 
Table 1. The results of the protocol on the sphere dataset. The original mesh consists of 720 vertices and 1436 triangles. An edge-detection step is indicated by ED, modified smoothing by S, and edge swapping by ES. Of the multiple smoothing steps only the first and last are shown in each case.

\begin{tabular}{|c|c|c|c|c|c|}
\hline Operatior & Average Min. Angle & Average Quality & Average Inaccuracy & Mean Radius & Variance \\
\hline Org & 35.41 & 0.76 & & 24.78 & 0.471 \\
\hline ED & 35.01 & 0.75 & 0.08 & 25.01 & 0.038 \\
\hline ES & 36.15 & 0.77 & & & \\
\hline $\mathrm{S}$ & 42.53 & 0.89 & & 24.82 & 0.012 \\
\hline $\mathrm{S}$ & \multicolumn{5}{|c|}{2 smoothing steps performed } \\
\hline S & 44.35 & 0.91 & & 24.27 & 0.016 \\
\hline ED & 44.49 & 0.91 & 0.121 & 24.98 & 0.049 \\
\hline ES & 44.73 & 0.91 & & & \\
\hline S & 46.16 & 0.92 & & 24.80 & 0.016 \\
\hline $\mathrm{S}$ & \multicolumn{5}{|c|}{5 smoothing steps performed } \\
\hline $\mathrm{S}$ & 47.37 & 0.93 & & 23.76 & 0.017 \\
\hline ED & 47.41 & 0.93 & 0.130 & 24.92 & 0.044 \\
\hline ES & 47.41 & 0.93 & & & \\
\hline
\end{tabular}

\section{Results}

The method was applied to $3 \mathrm{D}$ images of a sphere and CT images of a human wrist.

A grey scale volume $(100 \times 100 \times 100)$ containing a sphere of diameter 25 placed at the centre is used to test our method.

Table 1 shows the results from a run of the complete protocol. The table shows that the difference between each radius of the mesh after an edge-detection step (ED) and the real radius never exceeds 0.25 . Therefore, the vertices are placed within the precision of the edge detector. The convergence of modified smoothing steps (S) is slow, but several steps can be applied before ED is necessary. The variance after a modified smoothing step is lower than after ED. This can be attributed to the low-pass nature of smoothing and the high-pass nature of edge detection. Note that the variance after ED is a tenth of the initial variance. Figure 3 shows a histogram of the triangle quality of the mesh before and after processing by our method. Clearly, the method removes low-quality triangles and increases the amount of high-quality triangles. Similar results are obtained where the sphere was corrupted with additive Gaussian noise $(\sigma=2)$.

Next, we present some of the results obtained from a CT dataset of a human wrist. Four different metacarpal bones were processed: the os lunatum, the os scaphoid, the os triquetrum, and the os trapezium (see Figure 4). Table 2 shows the results of the protocol. From the table it follows that the quality obviously increases and the inaccuracy decreases for all bones. The os lunatum and the os scaphoid allow more modified smoothing steps until an edge detection is required. Figure 3 shows histograms of triangle quality of the os scaphoid and the os lunatum before and after processing. The histograms show a distinct improvement of the mesh quality.

\section{Conclusions}

We have created a high-quality scale-space edge detector that requires an initial approximation of the shape to extract and an upper bound estimate of the deviation from the actual object. A method to improve the mesh without generating geometric errors was shown. Both methods were succesfully coupled by the sequential application protocol. 

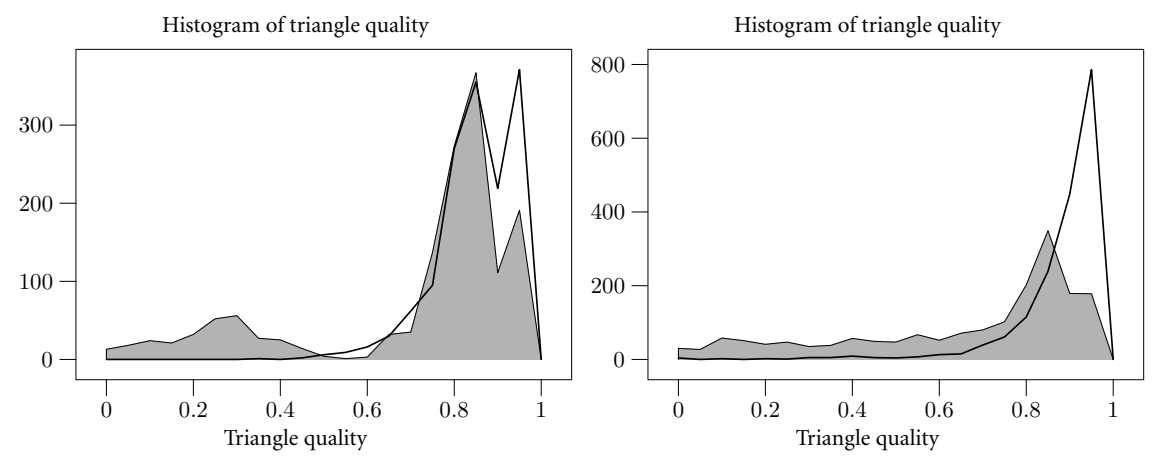

Fig. 3. Histograms of triangle quality ( $q_{\text {area }}$ criterion) before (solid grey) and after processing of a sphere with additive noise (left) and the Os Lunatum (right).

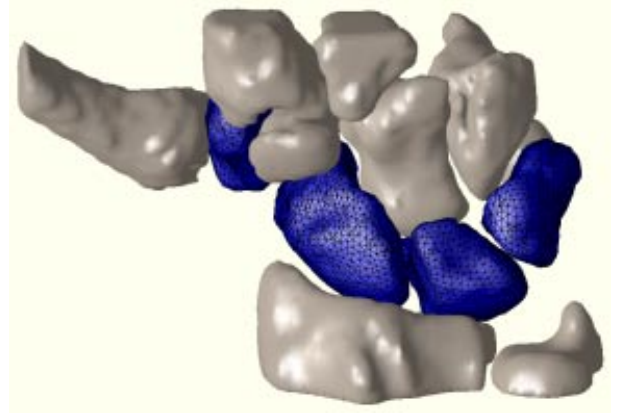

Fig. 4. A rendering of the wrist. The highlighted bones were processed by our method. From left to right the bones are: the os trapezium, the os scapoid, the os lunatum and the os triquetrum.

The meshes generated by our method are both accurate and consist of triangles of high quality. Apart from the initial mesh and the maximum deviation of this mesh, the only input required from a user is the desired quality of the mesh.

Further research will focus on adjusting the resolution of the mesh. Validation using phantom models in CT and MRI modalities is planned.

\section{References}

1. Babaud, J., Witkin, A., Baudin, M., And Duda, R. Uniqueness of the gaussian kernel for scale space filtering. IEEE Transactions on Pattern Analysis and Machine Intelligence 8, 1 (1986), 26-33.

2. Bank, R. E., ANd Smith, R. K. Mesh smoothing using A posteriori error estimates. SIAM Journal on Numerical Analysis 34, 3 (1997), 979-997.

3. Berzins, M. Mesh quality: A function of geometry, error estimates or both. In 7 th International Meshing Roundtable, Sandia National Lab (Oct. 1998), pp. 229-238.

4. Boender, E. Reliable delaunay-based mesh generation and mesh improvement. Communications in Numerical Methods in Engineering 10 (1994), 773-783.

5. Canny, J. A computational approach to edge-detection. IEEE Transactions on Pattern Anal$y$ sis and Machine Intelligence 8, 6 (1986), 679-697.

6. de Bruin, P., Vos, F., Post, F., Frisken-Gibson, S., and Vossepoel, A. Improving triangle mesh quality with surfacenets. In Medical Image Computing and Computer-Assisted Intervention - MICCAI (oct 200o), S. Delp, A. DiGioia, and B. Jaramaz, Eds. Third International Conference, Pittsburgh, PA, USA. 
Table 2. Results using the protocol on four metacarpal bones. Edge detection is indicated by ED, modified smoothing by S, and edge swapping by ES. For each ED step the inaccuracy is calculated. Note that the number of $S$ steps is different for some bones (indicated by $\downarrow$ ). For each bone the number of vertices and triangles is indicated.

\begin{tabular}{|c|c|c|c|c|c|c|c|c|c|c|c|c|}
\hline \multirow{2}{*}{\begin{tabular}{|l} 
Operation \\
Average $\rightarrow$
\end{tabular}} & \multicolumn{3}{|c|}{$\begin{array}{l}\text { Os Lunatum } \\
882 \mathrm{~V} \text { and } 1760 t\end{array}$} & \multicolumn{3}{|c|}{$\begin{array}{l}\text { Os Scaphoid } \\
1206 \mathrm{v} \text { and } 2408 \mathrm{t}\end{array}$} & \multicolumn{3}{|c|}{$\begin{array}{l}\text { Os Triquetrum } \\
668 \mathrm{v} \text { and } 1368 \mathrm{t}\end{array}$} & \multicolumn{3}{|c|}{$\begin{array}{l}\text { Os Trapezium } \\
790 v \text { and } 1576 t\end{array}$} \\
\hline & $\begin{array}{l}\text { Min. } \\
\text { Angle }\end{array}$ & Quality & Inacc. & $\begin{array}{l}\text { Min. } \\
\text { Angle }\end{array}$ & Quality & Inacc. & $\begin{array}{l}\text { Min. } \\
\text { Angle }\end{array}$ & Quality & Inacc. & $\begin{array}{l}\text { Min. } \\
\text { Angle }\end{array}$ & Quality & Inacc. \\
\hline Org & 31.41 & 0.69 & & 31.54 & 0.68 & & 30.92 & 0.67 & & 31.41 & 0.68 & \\
\hline ED & 31.64 & 0.69 & 0.27 & 31.73 & 0.69 & 0.30 & 31.40 & 0.69 & 0.36 & 31.58 & 0.69 & 0.65 \\
\hline ES & 33.02 & 0.71 & & 33.36 & 0.71 & & 32.77 & 0.71 & & 33.61 & 0.72 & \\
\hline $\mathrm{S}$ & 41.88 & 0.87 & & 42.88 & 0.88 & & 41.81 & 0.87 & & 42.86 & 0.88 & \\
\hline $\mathrm{S}$ & 43.16 & 0.88 & & 44.12 & 0.90 & & $\downarrow$ & $\downarrow$ & & $\downarrow$ & $\downarrow$ & \\
\hline ED & 43.37 & 0.89 & 0.18 & 44.27 & 0.90 & 0.16 & 41.96 & 0.87 & 0.20 & 42.89 & 0.88 & 0.29 \\
\hline ES & 43.51 & 0.89 & & 44.36 & 0.90 & & 42.13 & 0.87 & & 43.04 & 0.88 & \\
\hline $\mathrm{S}$ & 45.05 & 0.90 & & 45.89 & 0.91 & & 44.71 & 0.90 & & 45.67 & 0.91 & \\
\hline $\mathrm{S}$ & 45.42 & 0.91 & & 46.28 & 0.92 & & 45.36 & 0.91 & & $\downarrow$ & $\downarrow$ & \\
\hline ED & 45.57 & 0.91 & 0.18 & 46.38 & 0.92 & 0.16 & 45.45 & 0.91 & 0.19 & 45.46 & 0.91 & 0.26 \\
\hline ES & 45.57 & 0.91 & & 46.38 & 0.92 & & 45.48 & 0.91 & & 45.45 & 0.91 & \\
\hline
\end{tabular}

7. Florack, L., Romeny, B. T. H., Koenderink, J., And Viergever, M. Scale and the differential structure of images. Image and Vision Computing 10 (1992), 376-388.

8. Freitag, L. A. On combining laplacian and optimization-based mesh smoothing techniques. In AMD Trends in Unstructured Mesh Generation (1997), vol. 220, ASME, pp. 37-43.

9. Frey, W. H., ANd Field, D. A. Mesh relaxation: A new technique for improving triangles. International Journal for Numerical Methods in Engineering 31 (1991), 1121-1133.

10. Gibson, S. Constrained elastic surfacenets: generating smooth surfaces from binary sampled data. In Proceedings Medical Image Computation and Computer Assisted Interventions, MICCAI'98 (1998), pp. 888-898. http: //www.merl.com/reports/TR99-24/.

11. Lobregt, S., ANd Viergever, M. A. A discrete dynamic contour model. IEEE Transactions on Medical Imaging 14, 1 (Mar. 1995), 12-24.

12. Lorensen, W., ANd Cline, H. Marching cubes: a high resolution 3 D surface construction algorithm. In Proc. ACM SIGGRAPH'87 (July 1987), pp. 163-169.

13. Mcinerney, T., and Terzopoulos, D. Deformable models in medical image analysis: A survey. Medical Image Analysis 1, 2 (1996), 91-108.

14. Pardo, J., Cabello, D., and Heras, J. A snake for model-based segmentation of biomedical images. Pattern Recognition Letters, 18 (1997), 1529-1538.

15. SNel, J. G. Wrists in Space, Deformable models for segmentation and matching techniques for registration of $3 D M R$ and $C T$ images of the wrist. PhD thesis, Universiteit van Amsterdam, 2000.

16. Wit кin, A. Scale space filtering. In Proceedings, International Joint Conference on Artificial Intelligence (1983), pp. 1019-1023. 